\title{
LA POLAROGRAPHIE A IMPULSIONS, METHODE ANALYTIQUE EN RADIOPROTECTION
}

\author{
Yves CHAPRON et Henri FRANÇOIS \\ Service Technique d'Etudes de Protection - Dosimétrie \\ Centre d'Etudes Nucléaires de Fontenay-aux-Roses
}

\begin{abstract}
RÉSUMÉ
La radioprotection nécessite l'intervention de méthodes analytiques très fines. Les auteurs montrent que la polarographie à impulsions est une méthode de choix. Une première partie de l'exposé suivant décrit ce qu'est la polarographie à impulsions et indique ses limites et ses extensions. Dans la deuxième partie, les auteurs développent une extension de la méthode. Les résultats permettent l'analyse de l'uranium à partir de la concentration de ro parties par milliard.
\end{abstract}

\section{I - LA POLAROGRAPHIE A IMPULSIONS}

\section{LA POLAROGRAPHIE CLASSIQUE}

La polarographie est une méthode électrochimique basée sur l'interprétation des courbes intensité-potentiel. C'est un moyen physicochimique d'investigation intéressant, mais c'est dans le domaine de la chimie analytique que la polarographie connaît l'application la plus importante.

Heyrovsky [I] en $19^{22}$ expliqua le premier l'allure des courbes polarographiques. C'est en r925 qu'Heyrovsky et Shikata [2] construisirent le premier polarographe à enregistrement automatique.

Le principe en est le suivant : La cellule de mesure contient la solution à analyser et deux électrodes immergées permettent d'imposer une tension électrique croissant proportionnellement avec le temps. On enregistre alors le courant d'électrolyse correspondant. La tension est appliquée entre une électrode de référence dont le potentiel reste constant et une électrode à goutte de mercure tombante, dont le potentiel pourra varier dans un domaine compris entre le potentiel d'oxydation du mercure et le potentiel de réduction de l'électrolyte support ou du solvant. La courbe courant-tension d'une solution contenant un élément électroréductible est indiquée figure $\mathrm{x}$.

Les renseignements obtenus sont, pour des conditions opératoires fixes, à la fois d'ordre qualitatif et quantitatif; qualitatif par la mesure du potentiel de demi-vague E $\mathrm{I} / 2$ spécifique d'un élément, quantitatif par la mesure d'un courant limite proportionnel à la concentration de l'espèce électroactive en solution.

Limites de la méthode :

a) Le pouvoir de résolution ne permet pas de distinguer deux espèces si celles-ci ont des potentiels de demi-vagues distants de moins de $200 \mathrm{mV}$; cette limite est due à la forme des polarogrammes. 
b) La sensibilité est limitée par l'existence d'un courant résiduel. La mesure du courant limite, qui est dû à la diffusion de l'espèce qui subit l'électrolyse, ne donne une grandeur précise que lorsque sa valeur est importante par rapport au courant résiduel. Il faut noter que le transport de masse peut s'effectuer également par convection et migration, mais ces phénomènes peuvent être négligés si la solution n'est pas agitée, si elle ne présente pas de gradient de température, et si elle contient un excès d'électrolyte indifférent. Le courant résiduel est la conséquence de la charge du condensateur que constitue la couche double formée au niveau du contact électrode-électrolyte.

Aussi le courant limite de diffusion est-il du même ordre de grandeur que le courant capacitif pour des concentrations voisines de $10^{-5}$ mole par litre.

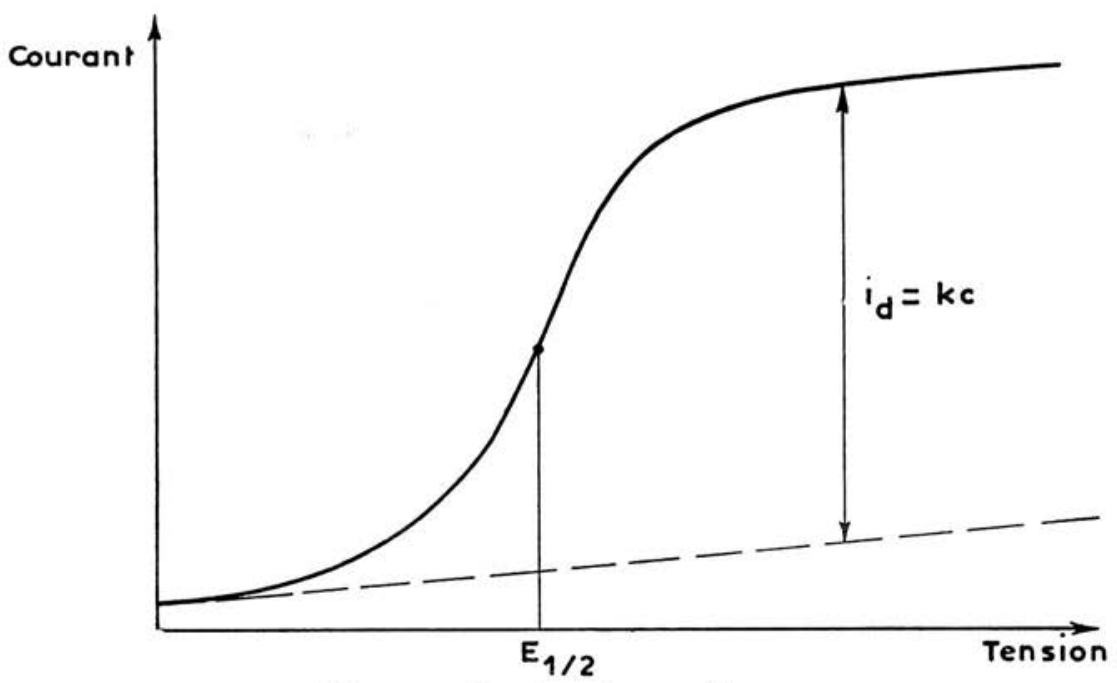

FIG. I. - Courbe polarographique.

AmÉliorations apPortéEs a la polarographie ClassiQue

\section{a) Polarographie dérivée}

L'introduction d'un circuit $\mathrm{RC}$ différentiateur dans le montage du polarographe [3] permet d'obtenir des polarogrammes dérivés. La vague classique est remplacée par une courbe en forme de pic dont l'amplitude est proportionnelle à la concentration de l'espèce électrolysée. Il est possible de mesurer quantitativement la concentration des éléments dont les pics sont au moins séparés par roo $\mathrm{mV}$. Il faut remarquer que la sensibilité n'est pas améliorée par ce montage.

\section{b) Polarographie oscillographique à potentiel imposé}

Le tracé des polarogrammes, c'est-à-dire des courbes intensité potentiel, s'effectue d'une façon continue. On impose la tension qui est une fonction linéaire du temps et l'on enregistre le courant correspondant. La relation entre 
la tension et le temps est du type $E=E_{1}+v t$. Lorsque $v$, la vitesse de balayage des tensions est faible (o,I V par minute) l'équation d'Ilkovic [4] donnant la valeur du courant de diffusion est de la forme $i_{d}=K n c$

$c$ : concentration de l'élément

$n$ : nombre d'électrons échangés

$K$ : constante de proportionnalité.

Lorsque $v$ devient important le courant est alors enregistré sur un oscilloscope et la vague obtenue initialement se transforme en un pic.

La relation de RandLes [s] permet d'exprimer le courant de pic en fonction de la vitesse de balayage des tensions, la relation est de la forme

$$
I p=K^{\prime} n^{3 / 2} v^{1 / 2} c
$$

$c$ : concentration de l'élément

$n$ : nombre d'électrons échangés

$K^{\prime}$ : constante de proportionnalité

$\nu$ : vitesse de balayage.

Il semblerait donc intéressant d'augmenter « $v$ ) pour assurer une plus grande sensibilité. En fait on montre que le courant capacitif est proportionnel à $($ $\nu)$, la sensibilité de la méthode s'en trouve donc amoindrie. A l'aide d'un circuit compensateur on peut réduire assez fortement le courant capacitif. Dans ce cas la limite analytique se situe aux environs de $10^{-7}$ mole par litre. Le pouvoir de résolution permet de distinguer deux espèces se déchargeant à des potentiels séparés par $100 \mathrm{mV}$.

\section{c) Polarographie à tension alternative surimposée}

C'est Mac Aleavy [6] qui le premier utilisa la polarographie en courant alternatif à des fins analytiques. Indépendamment BREYER [7] et ses collaborateurs entreprirent l'étude de la polarographie alternative dont le principe est le suivant :

On surimpose une tension sinusoïdale de faible amplitude (quelques $\mathrm{mV}$ ). Un système approprié n'enregistre que le courant résultant de la tension alternative. La courbe obtenue est proportionnelle à la dérivée des polarogrammes normaux. Elle présente donc un pic situé au niveau du potentiel de demi-vague qui permet par son amplitude de connaître la concentration de la substance electroactive.

Le courant maximum est de la forme [8].

$$
I_{\max }=K n^{2} f^{1 / 2} \Delta E C
$$

$K$ : constante de proportionnalité

$n$ : nombre d'électrons échangés

$f$ : fréquence de la tension alternative surimposée

$c$ : concentration de l'élément.

$\Delta E$ l'amplitude de la tension sinusoïdale surimposée doit être limitée à de faibles valeurs.

Le courant de pic étant proportionnel à la racine carrée de la fréquence, il semble possible d'augmenter la sensibilité en accroissant la fréquence. En fait 
il n'en est rien car dans ces conditions le courant capacitif croît plus rapidement que le courant du pic.

Pour augmenter la sensibilité de la polarographie en courant alternatif, BARKER et JENKINS [9] eurent l'idée de surimposer une tension non plus sinusoïdale mais carrée. Effectivement le condensateur que constitue la couche double peut être chargé et déchargé rapidement pendant que le courant dû à la réaction électrochimique décroît lentement. Dans ces conditions à l'issue d'un temps très court lorsqu'on enregistre le courant dû à la tension carrée, on constate que le courant de charge et de décharge de la double couche est faible devant le courant faradaïque dû à la réaction électrochimique. C'est ainsi que BARKER et JENKINS [9] n'enregistrèrent le courant que dans la $2^{\mathrm{e}}$ partie d'une alternance. L'expression du " courant de pic » est toujours une fonction de la fréquence [10]. Pratiquement on obtient les meilleurs résultats pour une fréquence de 225 cycles. La limite de sensibilité devient alors $4 \mathrm{IO}^{-8}$ mole/litre pour les systèmes réversibles et ${ }^{10^{-6}}$ mole/litre pour les systèmes irréversibles.

\section{Polarographie a impulsions}

Pour un système oxydo-réducteur reversible, ce n'est plus le courant de charge de la double couche qui limitera la sensibilité du polarographe à tension carrée, ni même le bruit de fond de l'électronique, mais un courant parasite qui naît dans le tube capillaire [II].

Lorsqu'un tube capillaire plonge dans une solution celle-ci en mouille la partie interne. Il se forme une mince pellicule de solution qui enrobe le mercure qui circule à l'intérieur. Lors d'une impulsion de tension il apparaît un courant de charge du condensateur formé par la double couche de la gaine cylindrique de solution. Ce courant parasite est difficile à compenser car il varie d'un capillaire à l'autre et est lié à son " histoire ». Le courant parasite que l'on appelle " réponse du capillaire » décroît plus vite que le courant faradaïque produit par le transfert de charge d'un couple oxydo-réducteur réversible. Puisque ces courants parasites capacitifs sont liés à la pulsation de la tension alternative, on peut les réduire par l'utilisation de très basses fréquences. En pratique, il faudrait donc utiliser des fréquences de l'ordre de 5 à ro cycles [ $\left.\mathrm{r}_{2}\right]$ par seconde, mais techniquement il est apparu plus intéressant d'utiliser des impulsions de potentiel.

\section{Distribution DES TENSIONS ET DES COURANTS EN POLAROGRAPHIE A IMPULSIONS}

Les impulsions ont une durée de 40 millisecondes et sont produites 2 secondes après la chute de la goutte précédente. Il n'y a qu'une impulsion par goutte de mercure.

\section{- Mode d'application de la tension d'électrolyse.}

Deux modes d'application de la tension sont possibles auxquels correspondent des polarogrammes normaux et dérivés.

- Si toutes les impulsions sont issues du potentiel de départ on obtient les polarogrammes normaux (fig. 2). 
- Les impulsions ont une amplitude constante, 35 ou $7 \mathrm{mV}$ et elles sont surimposées à la tension de polarisation qui est linéaire avec le temps (fig. 3).

Etablissement de la tension d'électrolyse en polarographie à impulsions.

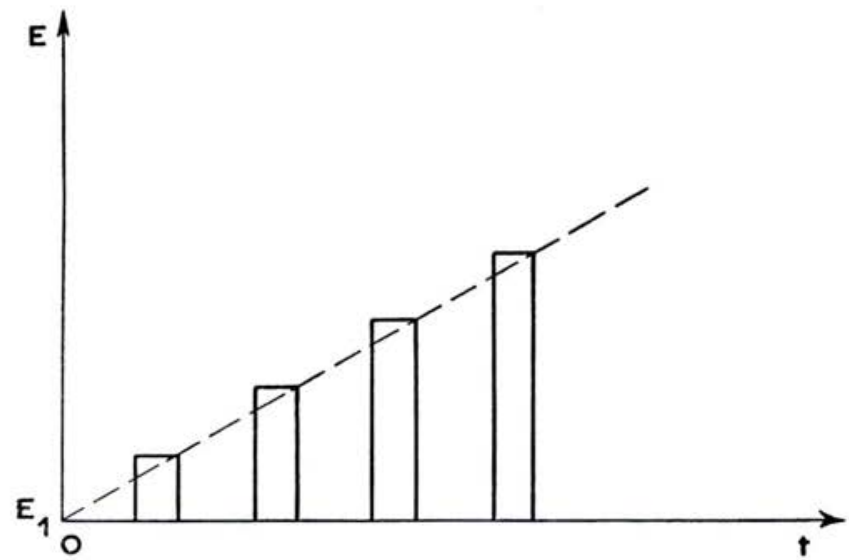

FIG. 2. - Programme de tension donnant des polarogrammes normaux.

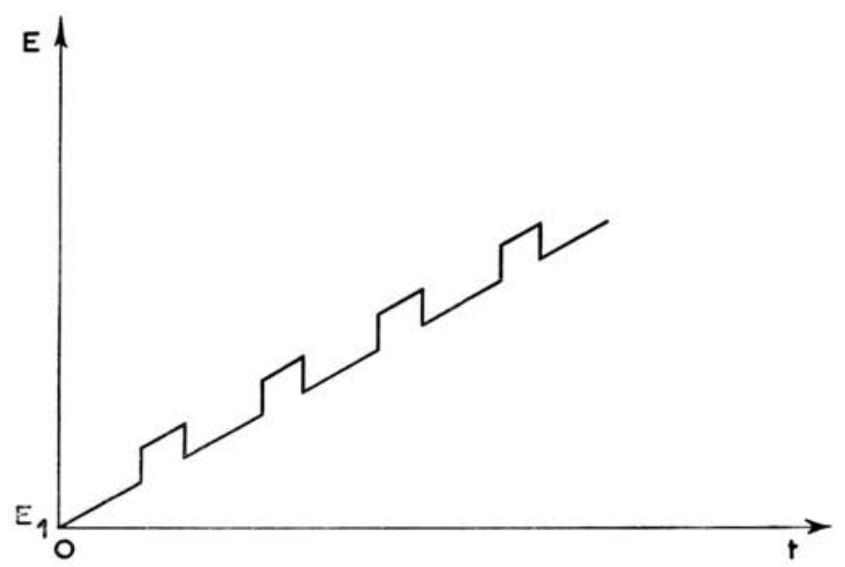

Fig. 3. - Programme de tension donnant des polarogrammes dérivés.

Dans ce cas on obtient des polarogrammes dérivés. La polarographie à impulsions dérivée est plus intéressante que celle qui conduit à des polarogrammes normaux. Nous n'utiliserons donc par la suite que la polarographie à impulsions dérivée.

\section{- Représentation des polarogrammes obtenus.}

Examinons la figure 4 .

Si l'on se place en $E_{1}$ le courant correspondant est $i_{1}$. Lorsqu'on superpose une impulsion $\Delta E$ le courant global passe de $i_{1}$ à $i_{1}+\Delta i$.

$\Delta i$ sera d'autant plus grand que la pente de la courbe sera élevée. 


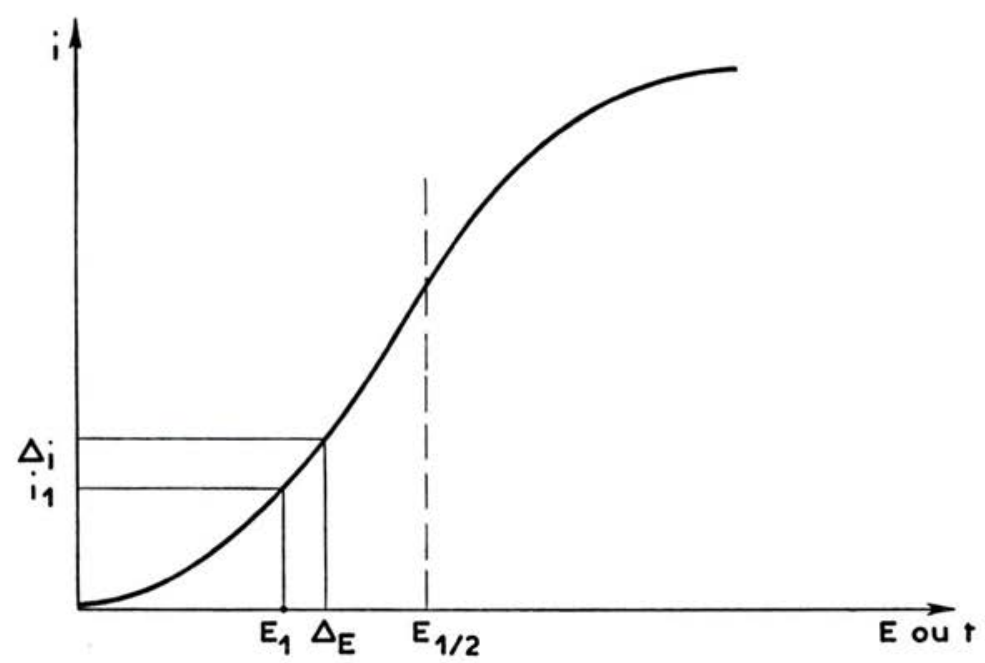

FIG. 4.

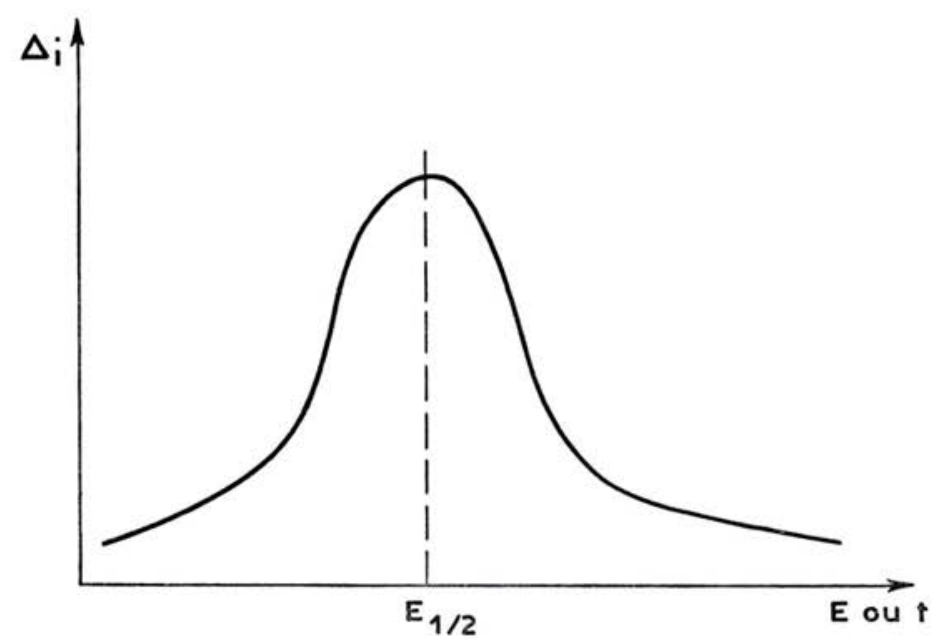

FIG. 5.

Polarogramme tracé avec impulsions d'amplitudes constantes.

$\Delta i$ sera maximum pour le potentiel de demi-vague $E_{\mathrm{I}} / 2$. La courbe $i=f(E)$ présentant pour cette valeur un point d'inflexion.

La courbe $\Delta i=f(E)$ aura donc une allure proportionnelle à la dérivée de la fonction $i=f(E)$ (fig. s). 
- Composition du courant transitoire résultant [I $\mathrm{I}]$ ].

De la même façon qu'en polarographie classique, on enregistre le courant faradaïque continu, établi par la composante continue et linéaire de la tension avec le temps.

Un montage composé de filtres permet d'éliminer ce courant et ne laisse passer que les courants transitoires qui apparaissent à la suite de l'impulsion de tension.

Le courant alternatif qui traverse le filtre est formé de deux composantes.

- Courant de charge et de décharge de la double couche du début et de la fin de l'impulsion.

- Courant faradaïque transitoire provenant de la variation de la cinétique d'électrode.

Seul le courant faradaïque nous intéresse, il faudra donc éliminer le courant capacitif. Au cours du temps ces deux courants décroissent différemment. Tandis que le courant capacitif décroît selon une loi exponentielle, le courant faradaïque décroît plus lentement.

L'expression du courant de charge est :

$$
I=\frac{\Delta E}{R} \quad \exp \left[\frac{-t}{R C}\right]
$$

$\Delta E$ est l'amplitude de l'impulsion de tension

$\mathrm{R} \quad$ la résistance globale du circuit électrolytique

C la capacité de la double couche qui se charge.

On peut remarquer que pour un temps de charge donné le courant s'annule d'autant plus vite que la résistance de l'ensemble est faible, et que l'amplitude de tension est petite.

En pratique, BARKER [II] mesure le courant moyen obtenu durant les 20 dernières millisecondes de l'impulsion. De cette façon le courant capacitif est négligeable.

Le courant enregistré correspond au courant faradaïque créé par l'impulsion de tension.

\section{Expression du courant faradaique transitoire}

Les relations donnant l'équation du courant pour les polarogrammes dérivés ont été établies par BARKER [II].

Cas d'un système reversible :

$$
\Delta i=\frac{n^{2} F^{2}}{R T} \cdot S \cdot C \Delta E\left(\frac{D}{\pi t}\right)^{1 / 2} \frac{P}{(\mathrm{I}+P)^{2}}
$$

$S$ : surface de l'électrode

$C$ : concentration de l'espèce en millimoles par litre

$D$ : coefficient de diffusion de la substance en $\mathrm{cm}^{2}$ seconde ${ }^{-1}$

$P \quad: \exp \left[\left(E-E_{1 / 2}+\frac{\Delta E}{2}\right) \frac{n F}{R T}\right]$

$E_{1 / 2}$ : potentiel de demi-vague du système considéré

$E$ : potentiel de l'électrode juste avant l'impulsion. 
$P$ est fonction du potentiel.

Pour $E=E_{1 / 2}-\frac{\Delta E}{2}$, nous avons $P=\mathrm{r}$; et $\frac{P}{(\mathrm{I}+P)^{2}}$ passe par un maximum pour cette valeur.

En conséquence, la fonction $\Delta_{t}=f(P)=f^{\prime}(E)$ est maximale.

L'expression de ce maximum est :

$$
\Delta_{i(\max )}=\frac{n^{2} F^{2}}{4 R T} \cdot S \cdot C \cdot \Delta E\left(\frac{D}{t \pi}\right)^{1 / 2}
$$

Nous voyons donc que le courant de pic $\left(\Delta_{i, \max }\right)$ est une fonction linéaire de la concentration, lorsque tous les autres paramètres restent constants.

BARKER a donné l'expression du courant faradaïque transitoire pour les systèmes faiblement réversibles et totalement irréversibles.

Les courants de pic à concentrations égales sont plus faibles; toutefois, ils sont plus importants qu'en polarographie à tension carrée.

L'auteur compare les résultats entre les deux méthodes et donne la relation suivante entre $\Delta_{i}(\max )$ pour les systèmes réversibles et irréversibles.

$$
\left(\frac{\Delta i \text { Irrev. }}{\Delta i \text { Rev. }}\right)_{\text {Impulsion }}^{=} 3,5\left(\frac{\Delta i \text { Irrev. }}{\Delta i \text { Rev. }}\right)_{\text {Tension carrée. }}
$$

Résultats obtenus avec le Polarographe à Impulsions - modèle MK II de la Southern and $\mathrm{C}^{\circ}$.

\section{- Limite de sensibilité}

Le constructeur donne la limite suivante : $10^{-8}$ mole par litre pour les systèmes réversibles. En fait, quelques éléments seulement sont mesurables à cette concentration (Cadmium et Plomb par exemple); d'autres, appartenant à des systèmes plus ou moins réversibles seront mesurables pour 4 à 5 10 ${ }^{-8}$ mole par litre, tels que Cuivre, Indium. En fait, le “ $E_{1 / 2}$ ) d'un élément ne sera pas étranger à la limite de sensibilité, car l'électrolyte indifférent présente un polarogramme en forme de berceau dont les deux branches montantes représentent l'oxydation ou la réduction de l'électrolyte ou du solvant. Ces deux branches ascendantes croissent selon une allure du type exponentiel et seuls les éléments dont le " $E_{1 / 2}$ ) se situe au fond du berceau ou au voisinage du fond, pourront être mesurés convenablement pour des concentrations voisines de $10^{-8}$ mole par litre, si le système auquel ils appartiennent est rapide. Les éléments se déchargeant à des potentiels placés très loin du fond du berceau sur la ligne de base ascendante, ne pourront pas être déterminés avec une aussi bonne précision.

En pratique, on pourra, par un choix judicieux soit trouver un milieu dans lequel le système intéressé sera rapide, soit un milieu dont le fond du berceau se trouvera plus près de $E_{1 / 2}$ du système, soit encore les deux à la fois pour disposer ainsi de la meilleure sensibilité.

\section{- Pouvoir de résolution de l'appareil et Interférences}

Deux termes fondamentaux interviennent : l'écart entre les potentiejs de demivague et le rapport des concentrations. 
La réversibilité des systèmes en présence et le nombre d'électrons intervenant dans leur décharge sont également importants. En effet, les systèmes irréversibles ont des pics étalés dans un domaine de potentiel plus ou moins grand et de ce fait empiètent sur les polarogrammes voisins.

Ainsi pour deux systèmes réversibles dont les potentiels de pic sont voisins de 200 millivolts, la limite qui permet encore une mesure de la concentration la plus faible est atteinte pour un rapport de concentration de 10000 .

Le pouvoir de résolution de l'appareil pour deux systèmes réversibles et de même concentration est voisin de 40 millivolts lorsqu'on utilise une amplitude d'impulsion de 7 millivolts.

\section{AMÉLIORATIONS DES POSSIBILITÉS ANALYTIQUES DE L'APPAREIL}

En dehors des moyens qui interviennent sur le milieu à analyser, on peut utiliser la redissolution anodique qui est une extension de la méthode [13].

Précisons tout de suite que cette méthode ne peut s'appliquer qu'aux seuls éléments dont le métal est soluble dans le mercure. Il faut également que le système auquel ils appartiennent soit suffisamment réversible.

On concentre sur une goutte de mercure pendante l'espèce à analyser, par électrolyse, puis on balaye le domaine de potentiel dans le sens positif. On oxyde de cette façon le métal dissous dans le mercure; la concentration préalable permet dans les cas heureux, d'obtenir un accroissement de la sensibilité d'un facteur voisin de roo.

Lorsque le métal dissous dans le mercure est plus noble que celui-ci, la méthode n'est pas utilisable, cependant, à l'aide d'électrodes solides peu corrodables en milieu aqueux, on peut utiliser la redissolution anodique pour certains métaux plus nobles que le mercure [14]. Le procédé a reçu lui-même une extension permettant d'accroître la sensibilité : la redissolution anodique dérivée. Cette dernière méthode a permis à PÉrone et BIrk [I $\mathrm{I}$ ] de détecter des ions $\mathrm{Cd}^{2+}$ à la concentration de 6.10 $10^{-11}$ mole/litre.

Ces résultats très intéressants nous conduisent à envisager l'utilisation de la redissolution anodique avec le polarographe à impulsion.

Il faut noter tout d'abord que l'appareil tel qu'il est ne permet pas d'utiliser les très fortes sensibilités car on ne peut compenser le niveau de base du polarogramme.

Au cours d'une série d'expériences nous avons utilisé les facteurs d'amplification $\frac{\mathrm{I}}{4}$ et $\frac{\mathrm{I}}{\mathrm{s}}$.

Les résultats peuvent être illustrés par l'exemple suivant, pour une concentration de $5 \mathrm{ro}^{-9}$ mole par litre de $\mathrm{Cd}^{2+}$ et un temps d'électrolyse de 2,5 minutes. Nous avons obtenu un pic de $2,5 \mathrm{~cm}$, ce qui laisserait espérer pour les espèces utilisables en redissolution anodique, une limite de $10^{-10}$ mole par litre à condition d'électrolyser pendant une demi-heure et d'utiliser la sensibilité maximum de l'appareil.

La polarographie à impulsion en redissolution anodique ne permet donc pas d'envisager de meilleurs résultats que la chronoampèrométrie linéaire dérivée par redissolution anodique. 


\section{- Utilisation des composés organométalliques pour l'analyse au polarographe à impulsions}

Nous avons vu les possibilités d'analyse très fine apportées par la polorographie à impulsions et nous avons noté que malgré les extensions, la méthode était réservée à quelques espèces privilégiées.

De nombreux éléments intéressants en radioprotection tels que uranium, plutonium, béryllium, thorium, terres rares, actinides, etc., ne peuvent pas se réduire en solution aqueuse à l'état métallique. Lorsqu'ils le peuvent les vagues polarographiques sont difficilement utilisables pour le dosage des traces. Les polarogrammes qu'ils donnent lorsqu'ils sont réductibles correspondent souvent à un changement de valence. On conçoit donc que ces divers éléments ne puissent pas être analysés par la redissolution anodique. Pratiquement pour obtenir une bonne sensibilité et une sélectivité généralement accrue, nous avons pensé que nous pourrions utiliser la formation de chélates ou complexes organométalliques. Nous développons la méthode dans le chapitre suivant.

BIBLIOGRAPHIE

[1] Heyrovsky, J. J. Chem. Listy 16, 256 (1922).

[2] Heyrovsky, J. \& Shikata, M. Rec. trav. Chim. 44, 496 (i925).

[3] LeVk̂Que, M.P. \& Roth, J. J. chim. phys. 46, 480 (1949).

[4] Ilkovic, D. Collection Czecboslov. cbem. Communs 6, 498 (1934).

[5] Randles, J.E.B. trans Faraday, soc. 44, 327 (1948).

[6] Mac. Alenvyc. Belgian Patent 443.003 (194I).

[7] Breyer, B. \& Gutmann, F. trans. Faraday. Soc. 42, 650 (1946).

[8] Delahay, P. New Instrumental Methods in electro-chemistry (1954), p. 170.

[9] Barker, G.C. \& Jenkins, I.L. Analyst 77, 685 (1952).

[ro] Barker, G.C. Faircloth, R.C. \& Gardner, A.W. AERE/C/R : 1786 (1958).

[ii] Barker, G.C. \& Gardner, A.W. AERE/C/R, 2297 (1958).

[12] Delahay, P. Rec. trav. chim. 67, 165 (1948).

[13] Maxwell, J.A. \& Graham, R.P. Chem. Revs 46, 47i (1950).

[14] Perone, S.P. Anal. Chem. 35, 2091 (1963).

[is] Perone, S.P. \& Birk, Anal. chem. 37, i-9 (ig65).

\section{II - UTILISATION DES COMPOSÉS ORGANOMÉTALLIQUES POUR L'ANALYSE DU POLAROGRAPHE A IMPULSIONS}

\section{INTÉRÊT DES COMPOSÉS ORGANOMÉTALLIQUES}

Ces corps présentent pour l'analyse polarographique l'avantage d'être sélectifs et de pouvoir céder davantage d'électrons lors de leur réduction électrochimique. De cette manière ou réalise une sorte d'amplification de la réduction du métal complexé, puisque les échanges d'électrons supérieurs à deux sont rares avec éléments minéraux. Les corps organiques peuvent dans certains cas libérer plus de dix électrons, lors de leur réduction. On voit tout de suite l'avantage d'un tel processus lorsque celui-ci est applicable. Il est inutile de travailler avec la haute sensibilité de l'appareil et les impuretés sont donc moins gênantes. Willard, H.H. et Dean, J.A. [r] furent les premiers à utiliser cette méthode. Ils l'appliquèrent à la formation d'un complexe coloré entre le solochrome violet 
et l'aluminium. Le solochrome seul se réduit à $-0,46$ volt par rapport à la nappe de mercure. En présence d'aluminium une nouvelle vague apparaît à - 0,64 volt. Cette vague est proportionnelle à la concentration d'aluminium présent dans la solution. Ainsi, le béryllium, métal voisin de l'aluminium n'interfère-t-il pas et la méthode a été utilisée pour doser l'aluminium dans le bérylium.

Depuis d'autres auteurs notamment en U.R.S.S. ont trouvé des relations analytiques à partir des polarogrammes de composés organométalliques ou organiques [2].

\section{A. Diverses possibilités d'adsorption}

La transposition à la polarographie à impulsion de la méthode utilisée en polarographie classique n'est possible que si aucun phénomène d'adsorption du colorant ne vient perturber les courbes de réduction. Si un phénomène d'adsorption se produit dans un domaine du potentiel non exploré, il n'y a pas de perturbation.

Toutefois, deux cas peuvent éventuellement permettre l'utilisation d'un colorant présentant une adsorption dans une zone de potentiel voisine de la zone étudiée.

a) La cinétique d'adsorption est très rapide et les 20 millisecondes de repos avant la mesure permettent au colorant de s'adsorber complètement. La mesure faite dans les 20 millisecondes suivantes n'enregistrera que le courant dû à une éventuelle réduction.

b) La cinétique d'adsorption est très lente et, pendant les 40 millisecondes que dure l'impulsion, on n'assiste qu'à une faible adsorption et les perturbations apportées sont négligeables par rapport au courant mesuré.

Cependant, il est évident qu'il faudra faire un choix entre les divers réactifs susceptibles d'être utilisés. Seuls ceux ne présentant pas de polarogrammes perturbés pourront être retenus.

De plus, pour des raisons de simplicité d'emploi, il est intéressant de trouver un réactif hydrosoluble aux concentrations utilisées. Il devra en être de même pour le complexe formé.

\section{B. Adsorption et réduction}

Nous allons voir qu'en présence d'une couche d'absorbat électroactif stable, la méthode est encore plus sensible. Supposons que le complexe s'adsorbe en couche monomoléculaire et obéisse ainsi à l'isotherme d'adsorption de Langmuir. Son expression est de la forme :

$$
m=\frac{m_{0} b P}{\mathrm{I}+b P} .
$$

Breyer et Bauer [3] ont donné l'expression du courant de pic en polarographie à vague carrée en présence d'une adsorption du type Langmuir; le courant du pic a pour expression

$$
i p=\frac{K a B C}{\mathrm{I}+2 B C}
$$

où $K$ contient l'ensemble des paramètres de la réaction électrochimique. 
$a$ : terme dépendant de la taille des molécules organique.

et

$$
B=\frac{b_{O} b_{R}}{b_{O}+b_{R}}
$$

où $b_{O}$ et $b_{R}$ sont les constantes d'adsorption de Langmuir de la forme oxydée et réduite.

$$
C=C_{O}+C_{R}
$$

concentration totale du complexe au sein de la solution.

Si l'on étudie des traces du composé on a l'inégalité

$$
\text { 2 } B C \ll \mathrm{I}
$$

et l'équation du courant du pic s'écrit

$$
i p=K a B C
$$

on obtient ainsi une relation linéaire entre ip et $C$.

On peut remarquer que $K a B$ représente la pente de la droite

$$
i p=f(C)
$$

et que celle-ci doit être nécessairement supérieure à

$$
K<B K a
$$

puisqu'il y a excès du composé à la surface de l'électrode.

Pour de fortes concentrations, on peut écrire

et la relation devient :

$$
\text { 2 } B C \gg \mathrm{I}
$$

$$
i p=\frac{K a}{2} .
$$

Dans ce cas, le courant de pic tend donc vers une valeur limite; la relation prend alors l'allure d'une hyperbole.

\section{Applications a L'Analyse}

Milner a analysé l'uranium dans l'eau de la Manche en utilisant un polarographe à impulsions. Toutefois la sensibilité est moins bonne que celle prévue puisque, en employant la sensibilité maximale de l'appareil, la limite de détection est de l'ordre de o,os à o, I p.p.m. d'UO ${ }_{2}^{2+}$ soit 2 à $4 \cdot 10^{-7} \mathrm{~m} / 1$. Milner a donc du concentrer ses échantillons.

Nous avons pensé que nous pourrions appliquer la polarographie au dosage de l'uranium, à l'aide de complexes organométalliques. Nous avons utilisé l'arsénazo qui est réactif colorimétrique de l'uranium [4]. 


\section{ETUDE EXPÉRIMENTALE}

\section{a) Effet de la dilution du complexant organique}

Une première phase du travail a été de comparer des polarogrammes tracés pour diverses concentrations d'arsenazo, pour repérer les meilleurs résultats analytiques. Nous avons donc opéré en milieu $\mathrm{HCl}$ o, I $N$; pour chaque concentration nous avons repéré les pics dus à l'uranium, et dans les cas positifs nous avons tracé un diagramme d'étalonnage.

\section{b) Résultats pour diverses dilutions}

Les concentrations des solutions d'arsénazo étudiées sont exprimées en moles par litre $(\mathrm{m} / \mathrm{l}): 10^{-3}, 10^{-4}, 10^{-5}, 10^{-6}$ (fig. $\left.\mathrm{I}, 2,3,4\right)$. Nous avons rassemblé pour les comparer les potentiels correspondant au maximum des pics dans le tableau $\mathrm{n}^{0} \mathrm{I}$.

TABLEAU I

\begin{tabular}{|c|c|c|c|}
\hline $\begin{array}{l}\text { Concentration } \\
\text { de l'arsenazo }\end{array}$ & Potentiel de pic/potentiel nappe $\mathrm{Hg}$ & $\mathrm{UO}_{2}{ }^{2+}$ & $\begin{array}{c}\text { Coefficient } \\
\text { d'amplification }\end{array}$ \\
\hline $10^{-3}$ & $-0,34,-0,46,-0,54,-0,72$ & $-0,26$ & $\underset{S 12}{I} \times \frac{I}{S}$ \\
\hline $10^{-4}$ & $-0,14,-0,30,-0,56$ & $-0,14$ & $\stackrel{I}{6} \times \stackrel{I}{5}$ \\
\hline $10^{-5}$ & $-0,10,-0,27,-0,54$ & $-0,27$ & ${ }_{16}^{1} \times{ }_{5}^{1}$ \\
\hline $10^{-6}$ & $*,-0,25,-0,50$ & $-0,25$ & $\begin{array}{l}\mathrm{I} \\
2\end{array} \times \frac{\mathrm{I}}{5}$ \\
\hline
\end{tabular}

* Le courant correspondant au " mur du solvant », par corrosion du mercure en présence de $\mathrm{Cl}^{-}$est important ou, du moins la sensibilité utilisée renvoie partiellement les pics sur la branche montante du diagramme. Le premier pic se trouve probablement à un potentiel $E<-0,10 \mathrm{~V}$ situé très loin vers le haut du diagramme et ne peut pas être distingué.

Pour expliquer que les polarogrammes d'un même composé diffèrent suivant sa dilution, on peut supposer que, cette fois, l'absorption obéit aux isothermes B.E.T., c'est-à-dire qu'il existe plusieurs couches d'absorption. Le nombre de ces couches peut varier avec la concentration de la substance absorbable et l'on imagine que les groupes moléculaires constitutifs des couches supérieures de l'absorption soient dans un état moins actif que les couches sous-jacentes.

L'électroréduction des couches externes d'absorption, nécessiteront la mise en jeu d'une énergie plus grande que l'électroréduction des couches internes. Les faibles concentrations limitent le nombre des couches de molécules absorbées et l'état d'activation plus grand, entraîne une réduction plus facile. Cette hypothèse pourrait expliquer que les potentiels de pic soient de moins en moins négatifs avec la dilution de l'espèce absorbable. Par ailleurs, l'isotherme 
B.E.T. tend vers l'isotherme de Langmuir aux faibles concentrations. On peut alors utiliser les relations :

$$
\text { ip }=f(C) \text {. }
$$

Relation ip $=f(U)$ pour les diverses concentrations de complexant:

Résultats des courants de pics obtenus pour diverses concentrations d'uranium et d'arsenazo.

$$
\begin{aligned}
& \text { Arsenazo : } \mathrm{Io}^{-3} \mathrm{~m} / \mathrm{l} \quad E p=-0, \mathrm{I} 6 \mathrm{~V} \\
& \text { Sensibilité : } \frac{\mathrm{I}}{\mathrm{SI} 2} \times \frac{\mathrm{I}}{\mathrm{s}} \text {. }
\end{aligned}
$$

\begin{tabular}{|c|c|c|c|c|c|c|c|c|c|c|c|}
\hline$U$ en $\mu \mathrm{g} / \mathrm{ml} \ldots \ldots \ldots$ & o & 6 & I 2 & I 8 & 24 & 30 & 36 & 42 & 48 & 54 & 66 \\
\hline Hauteur en $\mathrm{mm} \quad \ldots$. & - & 一 & 5 & 22 & 27 & 35 & 45 & 53 & 57 & 84 & 91 \\
\hline
\end{tabular}

TABLEAU 2

$$
\text { Arsenazo : } 10^{-4} \mathrm{~m} / \mathrm{l} \quad E p=-0,14 \mathrm{~V}
$$

Sensibilité : $\frac{\mathrm{I}}{64} \times \frac{\mathrm{I}}{5}$.

TABLEAU 3

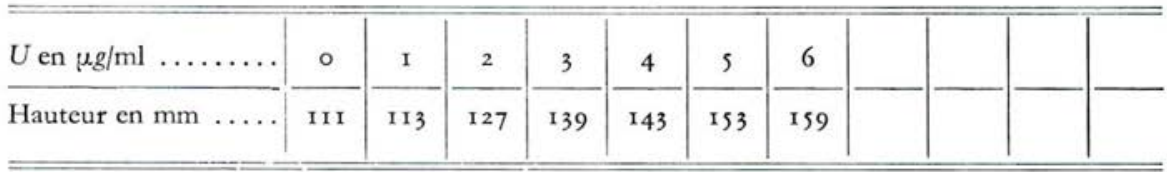

$$
\begin{aligned}
& \text { Arsénazo : }{ }_{10}^{-5} \mathrm{~m} / 1 \quad E p=-0,27 \mathrm{~V} \\
& \text { Sensibilité }=\frac{\mathrm{I}}{\mathrm{I} 6} \times \frac{\mathrm{I}}{\mathrm{s}} \text {. }
\end{aligned}
$$

TABLEAU 4

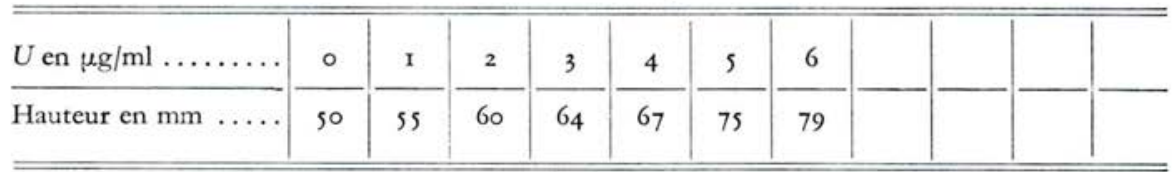

$$
\text { Arsénazo : }{ }_{10}^{-6} \mathrm{~m} / \mathrm{l} \quad E_{p}:-0,25 \mathrm{~V}
$$

Sensibilité : $\frac{\mathrm{I}}{2} \times \frac{\mathrm{I}}{\mathrm{S}}$.

TABLEAU s

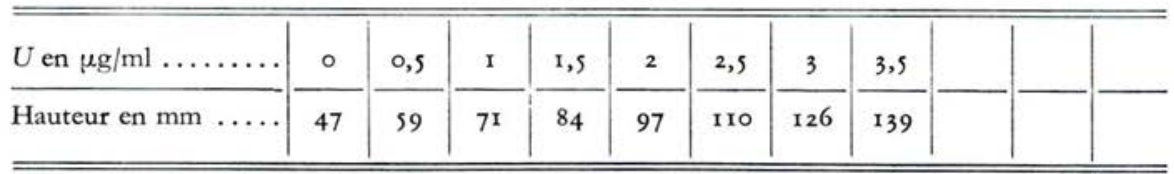




\section{c) Choix du milieu}

\section{Influence du $\mathrm{pH}$}

La sensibilité dépend du $p \mathrm{H}$ qui peut avoir une influence sur la formation du complexe et sur le nombre d'électrons mis en jeu lors de la réduction. Le $p \mathrm{H}$ détermine également la position des pics avec et sans uranium. Il s'agit donc de trouver un $p \mathrm{H}$ qui permette à la fois, la séparation d'un pic exclusivement dû à l'uranium du reste du polarogramme, et la meilleure sensibilité possible. Les résultats indiquent que c'est à un $p \mathrm{H}$ voisin de 7 que l'on obtient de bonnes caractéristiques. Le tampon réalisé avec de l'acétate de sodium donne les meilleurs résultats. La concentration optimum en arsénazo est $10^{-4} \mathrm{M} / 1$. Le pic maximum est obtenu après une heure d'attente. En utilisant le régime de gouttes " auto-trig " et l'amplification $\frac{\mathrm{I}}{\mathrm{I} 60}$ la concentration limite mesurable est de o, I p.p.m. d'uranium*. Cette limitation est due au fait que le pic de l'arsenazo trop voisin, masque celui du composé arsenazo-uranyl. Il nous a donc paru utile d'essayer différents $p \mathrm{H}$, autour de 7 , de façon à obtenir la sensibilité optimum. Pour mieux séparer les deux pics, nous utiliserons le régime de goutte « droptrig ", associé à un temps de balayage d'une heure d'exploration de potentiel (fig. 5, 6 et 7 )*.

Critères permettant de déterminer exactement le $p \mathrm{H}$ (fig. 10).

De façon à utiliser le milieu qui donnerait le meilleur résultat pratique, nous avons étudié les relations suivantes :

$$
i p=f(p \mathrm{H})
$$

et

$$
E p_{\text {arsenazo }}-E p_{\text {uranium }}=f(p \mathrm{H}) \text {. }
$$

Dans le domaine de $p \mathrm{H}$ compris entre 5,7 à 6,65 , nous avons réuni les résultats dans les tableaux suivants :

TABLEAU 6

\begin{tabular}{c|c|c|c}
\hline \hline \multicolumn{1}{c|}{} & 5,77 & 6 & 6,3 \\
\hline ip en mm ........ & 118 & 102 & 81,5 \\
\hline \hline
\end{tabular}

ip croît avec l'acidité.

\begin{tabular}{|c|c|c|c|}
\hline$p \mathrm{H}$ & 6 & 6,3 & 6,65 \\
\hline Eparsenazo $\ldots \ldots \ldots$ & $-0,56$ & $-0,57$ & $-0,55$ \\
\hline Epuranium .......... & $-0,63$ & $-0,66$ & $-0,72$ \\
\hline$\Delta E_{p} \ldots \ldots \ldots \ldots$ & 0,07 & 0,09 & 0,17 \\
\hline
\end{tabular}

TABLEAU 7

$\Delta E p$ décroît avec l'acidité. 
Le choix du $p \mathrm{H} 6,65$, relativement défavorable à l'amplitude du signal, permet en pratique la séparation des deux pics. Le défaut relatif de sensibilité est compensé par la possibilité de choisir des gammes d'amplification plus élevées.

Résultats obtenus pour $p \mathrm{H} \mathrm{6,6s} \mathrm{[s].}$

Les conditions opératoires diffèrent de celles utilisées à $p \mathrm{H}: 7$, par :

- " drop-trig » (I V en une heure),

- sensibilité $: \frac{\mathrm{I}}{8} \times \frac{\mathrm{I}}{\mathrm{s}}$.

En présence d'ions uranyle, un pic apparaît à $-0,705 \mathrm{~V}$ et les résultats sont les suivants (voir fig. 9 et ro).

TABLEAU 8

\begin{tabular}{l|c|c|c|c|c|c|c}
\hline \hline$U$ en $\mu \mathrm{g} / \mathrm{ml} . . . .$. & $\circ$ & 0,01 & 0,02 & 0,03 & 0,1 & 0,15 & 0,2 \\
\hline H en $\mathrm{mm}$ & 0 & 30 & 35 & 48 & II4 & 165 & 215 \\
\hline
\end{tabular}

\section{Conclusion}

La méthode permet de détecter to ppb d'uranium en régime de goutte tombante (“ drop-trig »), avec un balayage d'une heure et 0,1 à $0,2 \mathrm{ppm}$ en régime de chute automatique de gouttes (" auto-trig») avec un balayage d'un quart d'heure.

\section{BIBLIOGRAPHIE}

[1] Willard, H.H. \& Dean, J.A. Anal. chem., 22, i264 (1950).

[2] Tserkovnirskaya, J.A. \& Bykhovtseva, T.T. Zbur. Analiti. Kbimii, r9 5, 574-583 (1964).

[3] Breyer, B. \& BaUER, H.H. Alternating current polarography and Tensammetry (1963), Interscience publisber.

[4] Fritz, J.S. \& Johnson-Richard, D.M. Anal. Cbimi. Acta, 26 2, 164 (1959).

[s] Chapron, Y., Enrique, G. \& Francois, H. C.R. Ad Sc., t. 262, p. I 247 , 1966. 


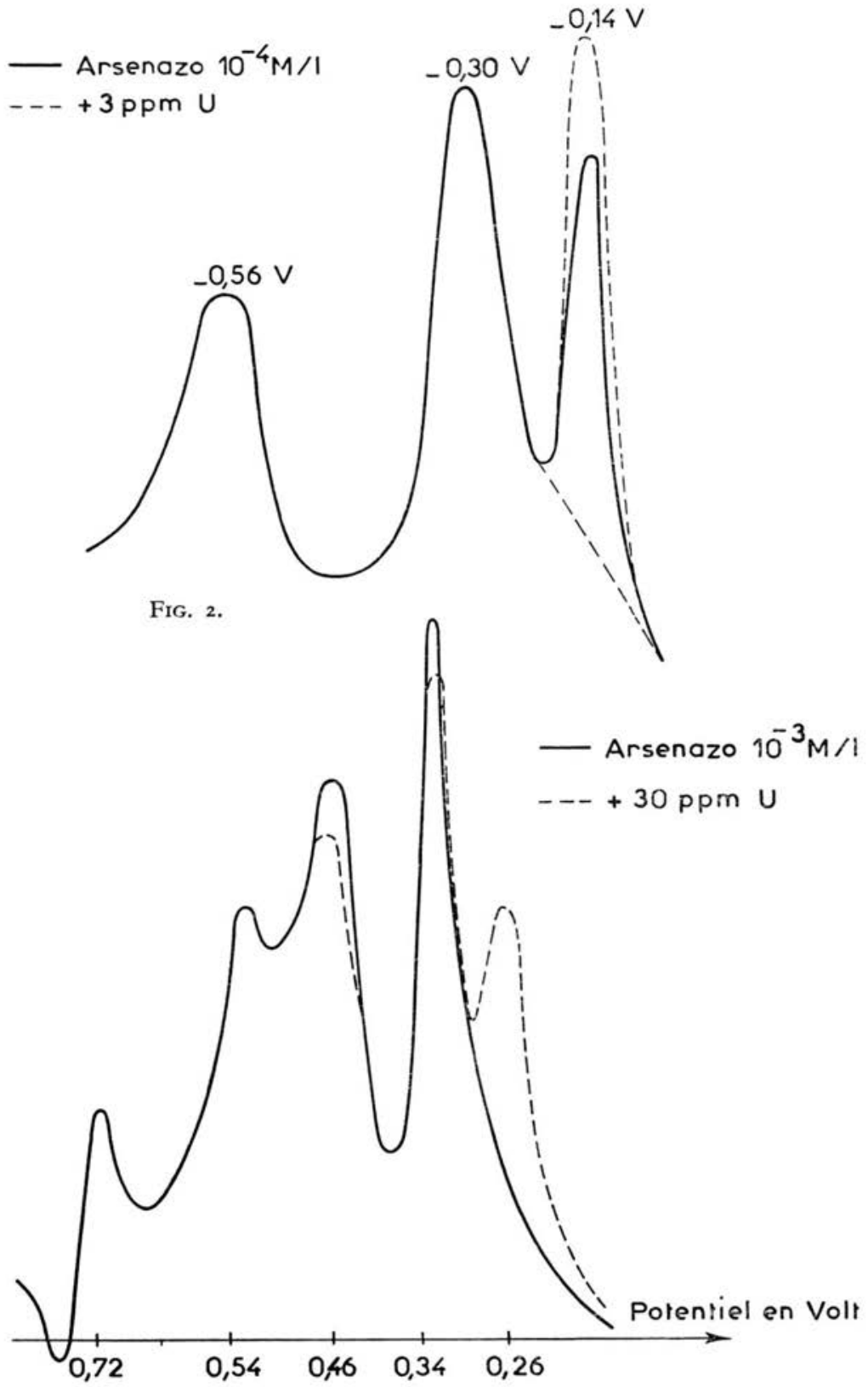

FIG. I. 
- Arsenazo $10^{-6} \mathrm{M} / \mathrm{I}$

---+2 pp $\mathrm{m}$ U

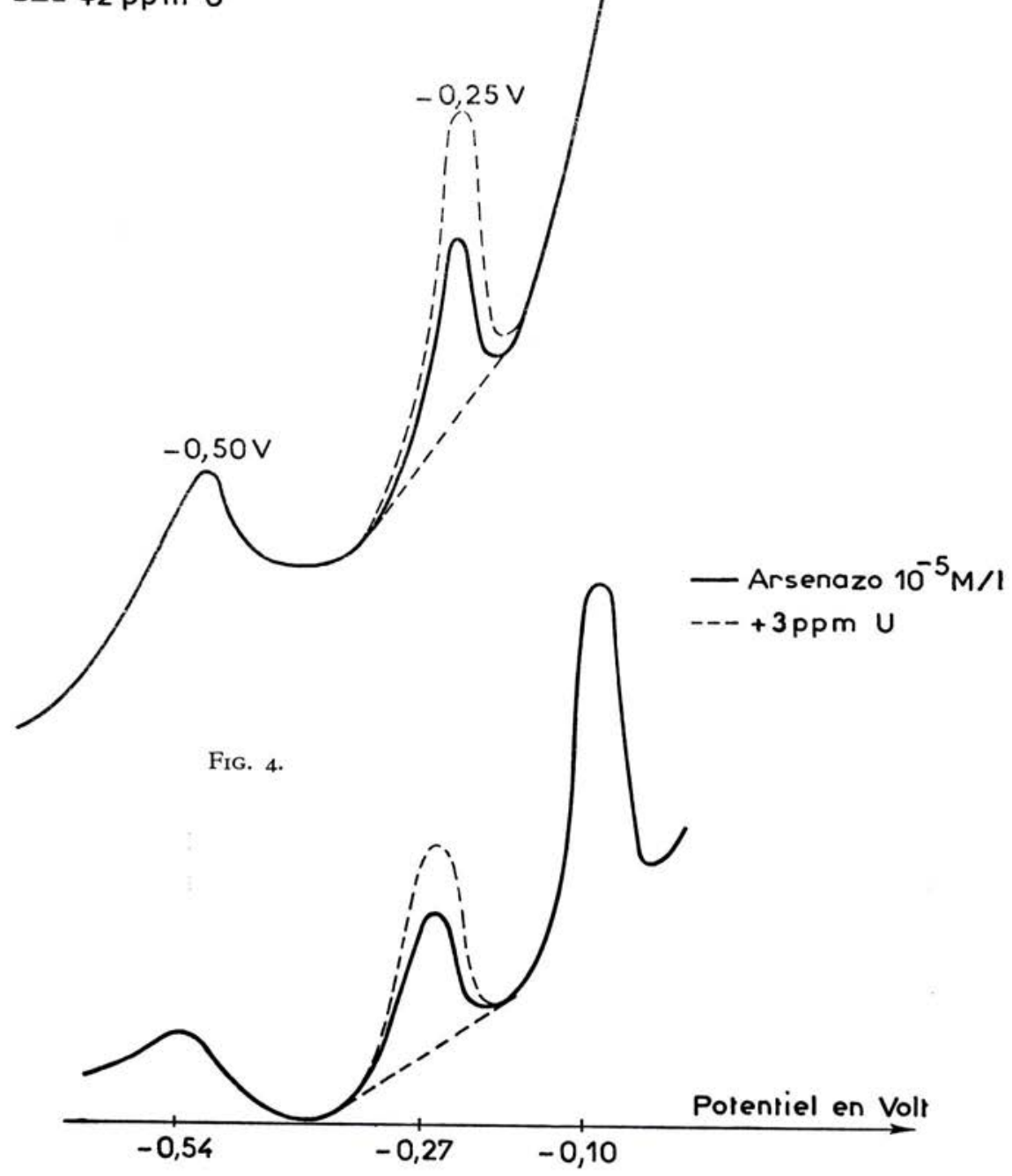

FIG. 3. 


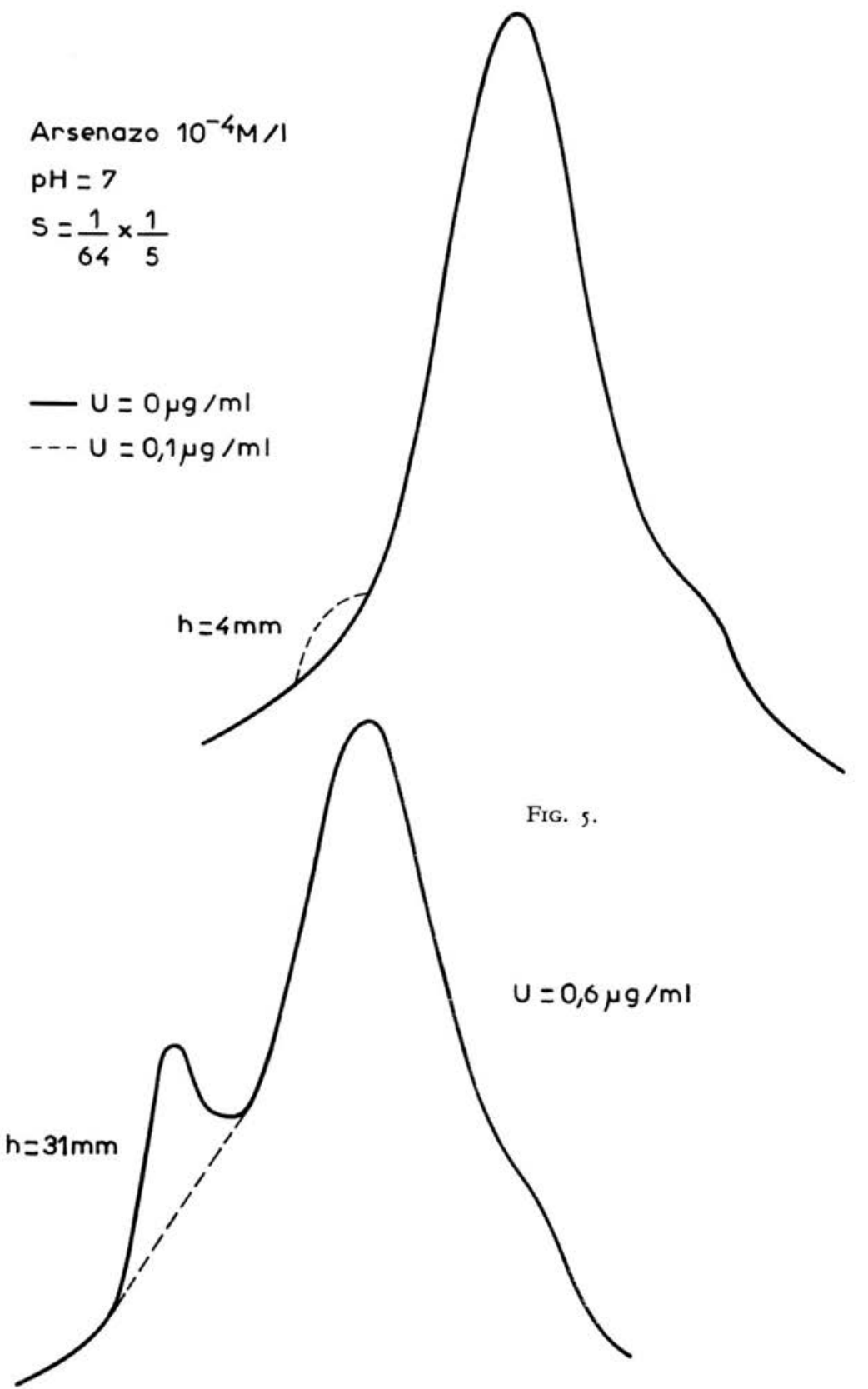

FIG. 6. 


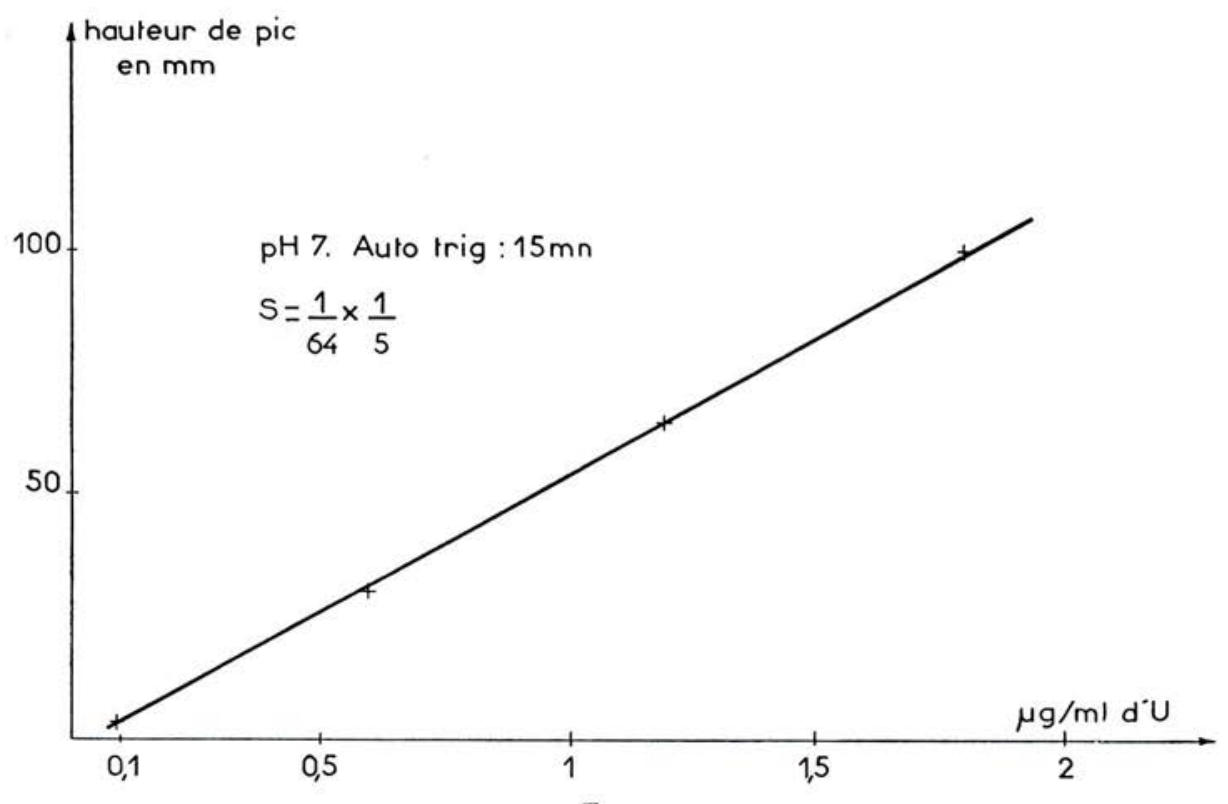

FIG. 7.

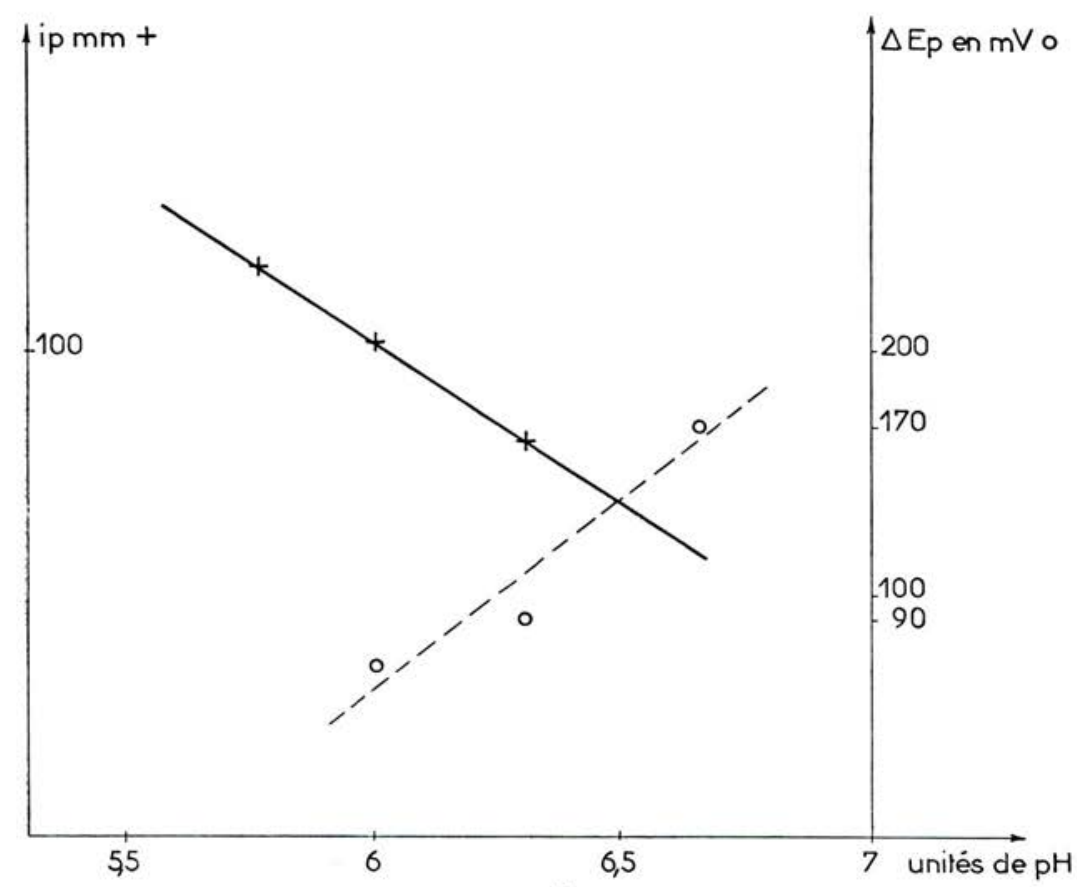

FIG. 8. 


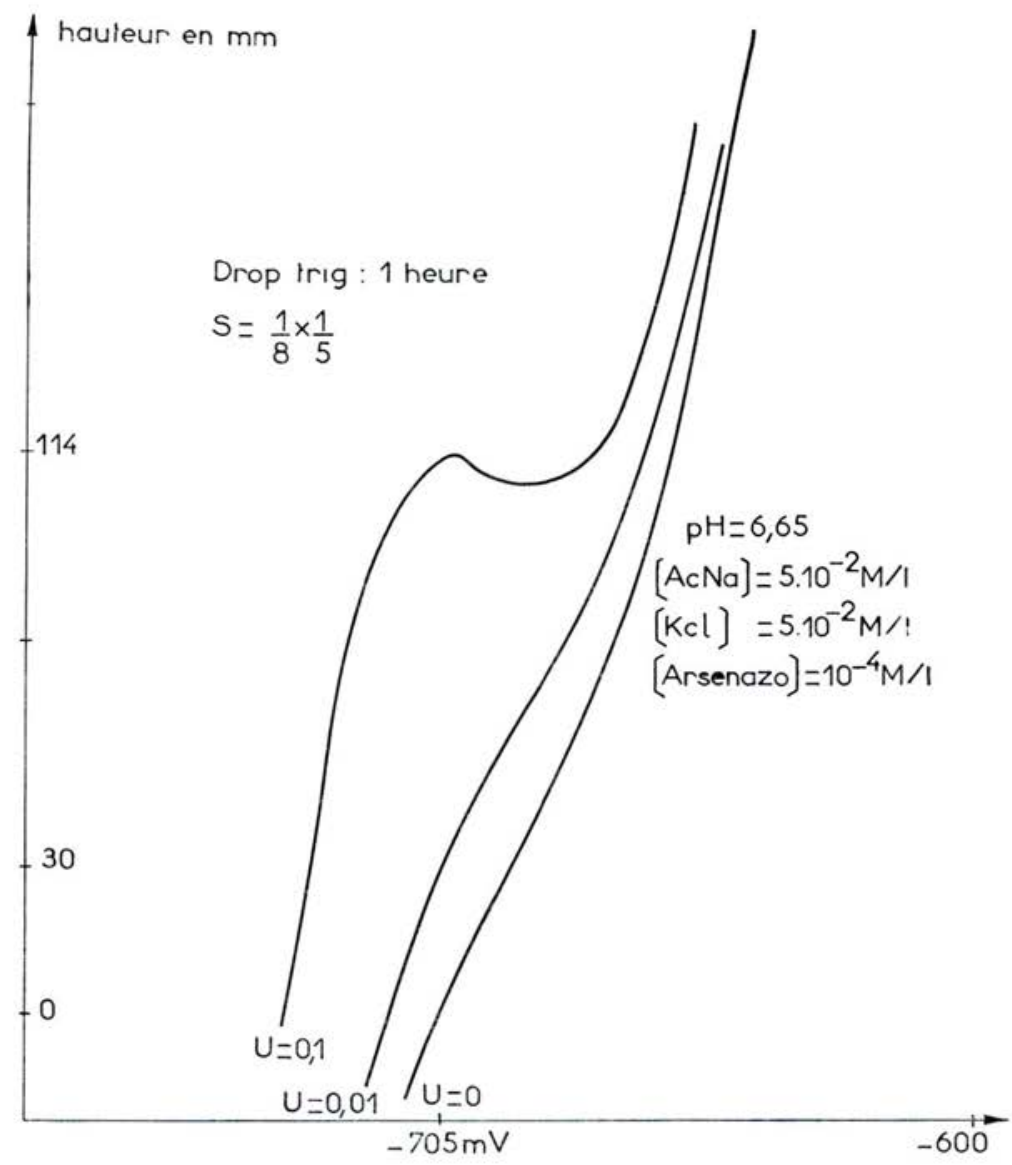

FIG. 9. 


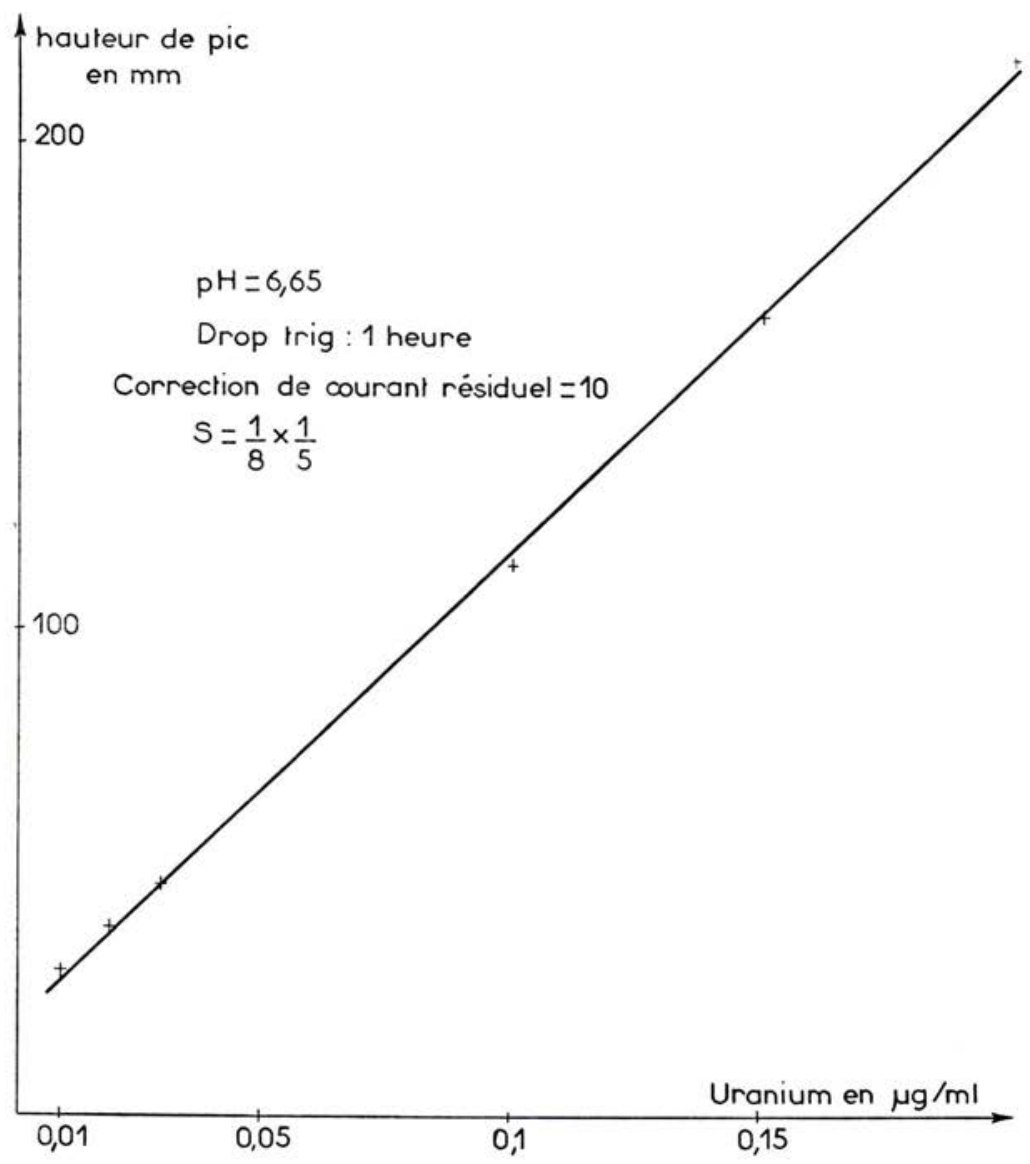

FIG. 10. 